\title{
Demonstration of Coherent Terahertz Transition Radiation from Relativistic Laser-Solid Interactions
}

\author{
Guo-Qian Liao ${ }^{1}$, Yu-Tong Li ${ }^{1,5 *}$, Yi-Hang Zhang ${ }^{1}$, Hao Liu ${ }^{1}$, Xu-Lei Ge ${ }^{2}$, Su Yang ${ }^{2}$, Wen-Qing \\ Wei $^{2}$, Xiao-Hui Yuan ${ }^{2,5^{*}}$, Yan-Qing Deng ${ }^{3}$, Bao-Jun Zhu ${ }^{1}$, Zhe Zhang ${ }^{1}$, Wei-Min Wang ${ }^{1,5}$, \\ Zheng-Ming Sheng ${ }^{4,2,5}$, Li-Ming Chen ${ }^{1}$, Xin Lu ${ }^{1}$, Jing-Long Ma ${ }^{1}$, Xuan Wang ${ }^{1}$, and Jie Zhang ${ }^{2,5}$ \\ ${ }^{1}$ Beijing National Laboratory for Condensed Matter Physics, Institute of Physics, Chinese \\ Academy of Sciences, Beijing 100190, China \\ ${ }^{2}$ Key Laboratory for Laser Plasmas (MoE) and Department of Physics and Astronomy, Shanghai \\ Jiao Tong University, Shanghai 200240, China \\ ${ }^{3}$ College of Science, National University of Defense Technology, Changsha 410073, China \\ ${ }^{4}$ SUPA, Department of Physics, University of Strathclyde, Glasgow G4 ONG, UK \\ ${ }^{5}$ Collaborative Innovation Center of IFSA (CICIFSA), Shanghai Jiao Tong University, Shanghai \\ 200240, China
}

Coherent transition radiation in the terahertz $(\mathrm{THz})$ region with energies of sub-mJ/pulse has been demonstrated by relativistic laser-driven electron beams crossing the solid-vacuum boundary. Targets including mass-limited foils and layered metal-plastic targets are used to verify the radiation mechanism and characterize the radiation properties. Observations of $\mathrm{THz}$ emissions as a function of target parameters agree well with the formation-zone and diffraction model of transition radiation. Particle-in-cell simulations also well reproduce the observed characteristics of $\mathrm{THz}$ emissions. The present $\mathrm{THz}$ transition radiation enables not only a potential tabletop brilliant $\mathrm{THz}$ source, but also a novel noninvasive diagnostic for fast electron generation and transport in laser-plasma interactions.

PACS numbers: 52.38.Kd, 52.59.Ye, 41.60.Dk

*To whom correspondence should be addressed. Email: ytli@iphy.ac.cn and xiaohui.yuan@sjtu.edu.cn 
High power terahertz (THz) sources can serve as a unique tool [1] in the exploration of condensed-matter dynamics [2,3], biomedical imaging [4], and wireless communications [5]. Over the last decades relativistic electron beams from conventional accelerators have been applied to generate strong $\mathrm{THz}$ radiation through various mechanisms such as transition radiation [6], Cherenkov radiation [7], synchrotron radiation [8], diffraction radiation [9], etc. The energy of THz pulses through transition radiation in linear accelerators has reached $\sim 600 \mu \mathrm{J} /$ pulse [10].

Relativistic electron beams can also be generated in the interactions of intense laser pulses with low-density gas or high-density solid targets. For gas targets, electrons can be accelerated to a GeV energy level with wakefields $[11,12]$. With such an electron beam, Leemans et al. have observed a $\sim 0.3 \mu \mathrm{J} \mathrm{THz}$ pulse through transition radiation [13]. Strong $\mathrm{THz}$ radiation from laser-solid interactions has also been demonstrated [14,15,16,17]. Compared with the cases of gas targets, electron beams from solid targets have much higher charge, up to $\mathrm{nC}-\mu \mathrm{C}$. Recently $\mathrm{THz}$ radiation with energies of $>700 \mu \mathrm{J}$ has been reported from the rear surface of a foil target $[18,19]$. Since the $\mathrm{THz}$ yield is found to be correlated with the square of the proton number, it is attributed to a transient dipole-like charge distribution structure formed by target normal sheath acceleration (TNSA), referred to hereafter as TNSA radiation [18]. On the other hand, in principle, THz radiation can be produced efficiently via the transition radiation process at the rear surface of a laser-irradiated thin solid foil [20], since both the short electron bunch duration and high bunch charge are ideal for this mechanism. Previous studies on the transition radiation from solid targets mainly concern in the optical region $[21,22]$. While the radiation in the THz regime has so far not yet been verified experimentally.

In this Letter, we will report the experimental demonstration of $\mathrm{THz}$ emissions via coherent transition radiation (CTR) when the laser-produced electron beams pass through the rear solid-vacuum interface. Various target structures and parameters are adopted to characterize the $\mathrm{THz}$ radiation properties, which can be well explained by the theoretical model of CTR.

The experiment was carried out on the laser system at the Laboratory for Laser Plasmas, 
Shanghai Jiao Tong University. Figure 1 shows the layout of the experimental setup. A 2 J, 30 fs, $800 \mathrm{~nm}$-polarized laser pulse was focused onto solid targets at an incidence angle of $54^{\circ}$ with a peak irradiance of $\sim 1.5 \times 10^{19} \mathrm{~W} / \mathrm{cm}^{2}$. The laser contrast ratio in the ns range was $\sim 10^{-5}$. The $\mathrm{THz}$ emissions were collected at $75^{\circ}, 45^{\circ},-60^{\circ}$ and $-75^{\circ}$ with respect to the target normal, and then refocused into cross-calibrated pyroelectric detectors. High-resistance silicon wafers were placed in front of $\mathrm{THz}$ detectors to block the visible light. The spectrum and polarization of $\mathrm{THz}$ radiation at $-75^{\circ}$ were measured by a set of pre-calibrated low-pass or band-pass filters and a $\mathrm{THz}$ polarizer, respectively.

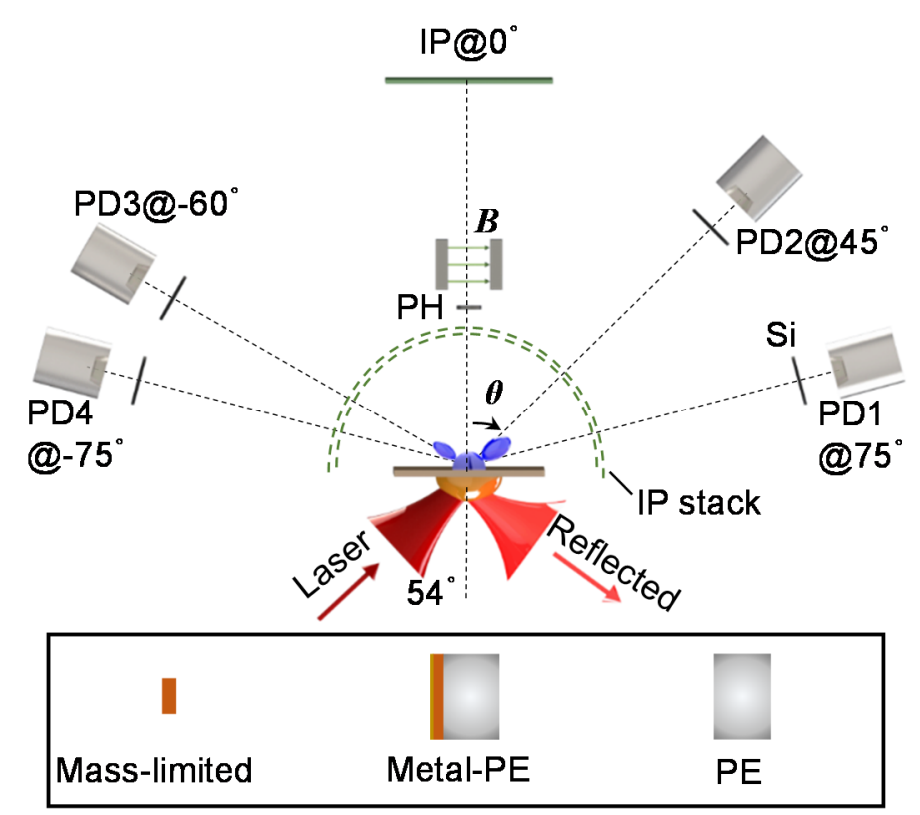

FIG. 1. Schematic of the top view of the experimental setup. The THz collection lens and windows are not shown. The lower inset sketches the three types of targets used. PD: pyroelectric detector; PH: pinhole; PE: polyethylene.

The TNSA ions were detected by an ion energy spectrometer aligned in the target rear normal $\left(0^{\circ}\right)$. Image plate (IP) was used as the ion detector. In some shots, the angular distribution of the forward electrons was measured by a double-layered IP stack surrounding the target. The IP stack was wrapped with $\mathrm{Al}$ filters to shield from the visible light, ions and low-energy x-ray photons.

Three types of targets were used in the experiment, polyethylene (PE), mass-limited and metal-PE targets. Mass-limited targets were $5 \mu \mathrm{m}$ thick copper $(\mathrm{Cu})$ foils with different sizes. The metal-PE target consisted of three layers, a $1 \mu \mathrm{m}$ thick gold, a $4 \mu \mathrm{m} \mathrm{Cu}$, and a PE layer with 
different thickness in the range $0-500 \mu \mathrm{m}$.

Figure 2(a) shows the typical frequency spectrum of $\mathrm{THz}$ radiation at $-75^{\circ}$ from the metal foils without the PE layer. The THz radiation covers a broad range up to $30 \mathrm{THz}$, which is dominated by the low frequency component $(<3 \mathrm{THz})$. Figure 2(b) shows the dependence of the measured $\mathrm{THz}$ intensity on the polarizer angle and the fitting to cosine-squared function. The radiation is elliptically polarized, mainly $p$-polarized but also with $s$-polarized components. The spectral and polarization characteristics indicate that the observed emission is not thermal.

Blue circles in Fig. 2(c) show the typical angular distribution of the measured $\mathrm{THz}$ radiation from the metal foils. The $\mathrm{THz}$ radiation at $0^{\circ}$ was measured by replacing the ion energy spectrometer with a set of $\mathrm{THz}$ detection system. It shows an asymmetric "double-wing-like" distribution. The $\mathrm{THz}$ intensity at $\pm 75^{\circ}$ is much higher than that at $0^{\circ}$, and the radiation at $75^{\circ}$ is stronger than that at $-75^{\circ}$.

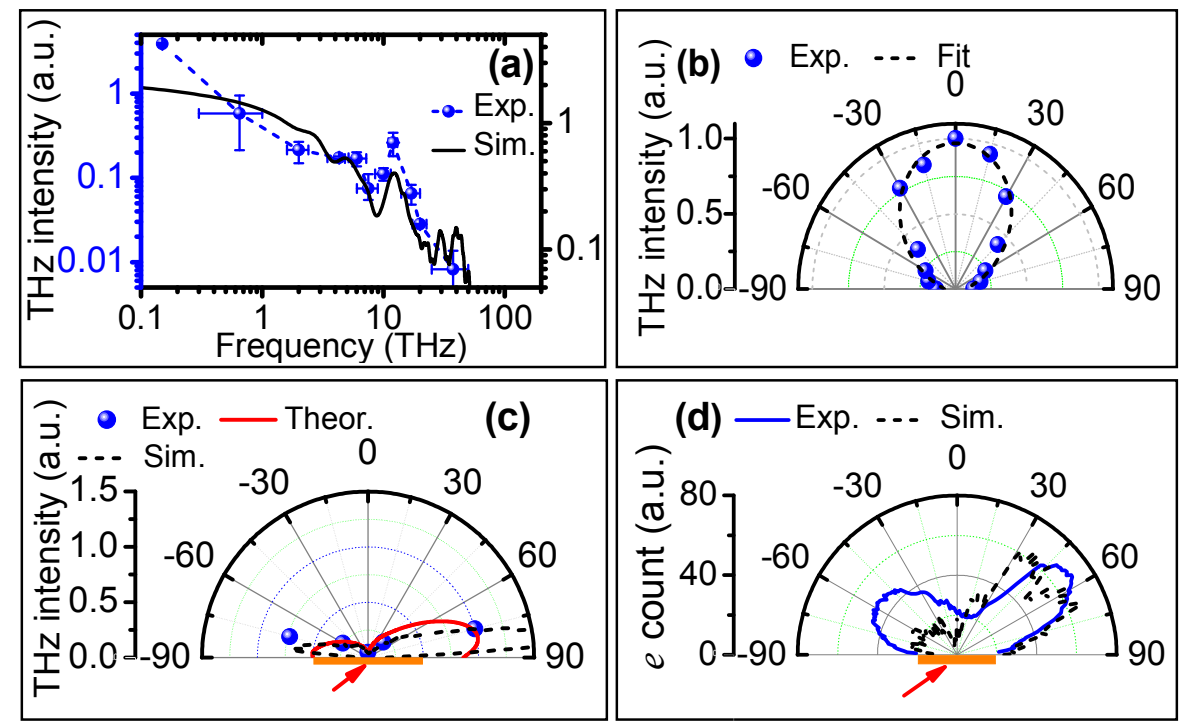

FIG. 2. (a) Experimentally measured (blue circle dashed) and simulated (black solid) frequency spectra of the THz emission at $-75^{\circ}$ from the metal foils without the PE layer. (b) Measured $\mathrm{THz}$ intensity (blue circle) as a function of the THz polarizer angle and the corresponding fitting curve (black dashed) with the cosine-squared function. (c) $\mathrm{THz}$ radiation angular distributions obtained from the experiment (blue circle), numerical simulation (black dashed) and theory model (red solid), which are normalized by the $\mathrm{THz}$ intensity at $75^{\circ}$. (d) Fast electron angular 
distributions obtained from experimental measurements (blue solid) and numerical simulations (black dashed). The red arrows in (c) and (d) indicate the laser incidence.

To understand the $\mathrm{THz}$ radiation and the generation of fast electrons, we have performed two-dimensional particle-in-cell (PIC) simulations using the code KLAPS [23]. In the simulations, a laser pulse with similar parameters to the experiment is incident onto a plasma slab with an exponential density increasing from $0.02 n_{\mathrm{c}}$ to $10 n_{\mathrm{c}}$ and a density scalelength of $L=3 \lambda_{0}$ or $6 \lambda_{0}$, where $n_{\mathrm{c}}$ is the critical density for the laser wavelength of $\lambda_{0}$. Since the simulated results with $L=3 \lambda_{0}$ and $6 \lambda_{0}$ are similar, here we only show the results of $L=3 \lambda_{0}$. A strong quasi-half-cycle electromagnetic emission is observed at the rear of the plasma slab. The simulated spectrum and angular distribution are shown in Fig. 2(a) and Fig. 2(c), respectively. Both of them are in agreement with the experimental results.

Figure 2(d) shows angular distributions for $>500 \mathrm{keV}$ fast electrons escaping from the target rear, which are measured in the experiment by the IP stack and obtained from numerical simulations, respectively. For the measured one, one peak is near $54^{\circ}$, in the direction of laser incidence. The other is near $-54^{\circ}$, in the opposite direction of laser reflection. Fitting with a cosine-squared function, we can obtain the divergence angles (FWHM) $\sim 35^{\circ}$ and $50^{\circ}$ for the electron beams at $54^{\circ}$ and $-54^{\circ}$, respectively. The PIC simulated distribution also has two peaks, which is similar to the experimental measurement. The simulations indicate that the peaks are mainly due to the ponderomotive acceleration of the incident and reflected laser pulses, respectively. The energy spectra of the electrons in simulations show a quasi-thermal distribution with a temperature of $\sim 1$ $\mathrm{MeV}$, in agreement with the scaling law of ponderomotive acceleration [24].

Several physical mechanisms based on laser-excited transient currents $[14,15,16]$ or plasma waves [17] have been proposed to explain the THz radiation from the front solid surface. However, they do not apply here. Since the forward fast electron beam can pass through the solid rear surface, the transition radiation could be responsible for the observed $\mathrm{THz}$ radiation from the rear surface [20]. According to the model of transition radiation [13], the wavelength range $\lambda$ of coherent transition radiation is predicted to be $\sigma_{\mathrm{ez}}<\lambda<\rho / \sqrt{\gamma^{2}-1}$, where $\sigma_{\mathrm{ez}}$ is the longitudinal length of 
electron beams, $\rho$ the target size, and $\gamma$ the relativistic Lorentz factor, respectively. In our case, electrons are mainly accelerated by the laser ponderomotive force, and the corresponding electron beam length $\sigma_{\mathrm{ez}} \sim c^{\cdot} \cdot \tau_{\mathrm{L}} \sim 10 \mu \mathrm{m}$, where $c$ is the light velocity in vacuum and $\tau_{\mathrm{L}}$ the laser pulse duration, respectively. Therefore the radiation should be mainly within $30 \mathrm{THz}$, which concurs with the experimental and simulated results.

The transition radiation will be elliptically polarized when the electrons are emitted out of the detection plane obliquely [25]. In principle, the polarization properties of transition radiation carry the information of the electron direction and divergence. By use of the measured degree of polarization $\sim 0.78$ for the $\mathrm{THz}$ radiation at $-75^{\circ}$, we infer the divergence angle of the electron beam at $-54^{\circ}$ to be $\sim 48^{\circ}$, which is consistent with the measured result. More details of the estimation are given in the Supplemental Material [26]. Using the measured electron angular distribution, we have calculated the $\mathrm{THz}$ radiation angular distribution, which is shown as the red line in Fig. 2(c). The calculated is also an asymmetric double-wing-like distribution, in agreement with the observation.

The model of transition radiation predicts that the radiation intensity is closely dependent on target parameters such as the size and dielectric property of targets. To verify those, we have investigated the $\mathrm{THz}$ radiation as a function of various target parameters systematically. Figure 3 shows the THz radiation from the $5 \mu \mathrm{m}$ thick mass-limited $\mathrm{Cu}$ targets with different sizes. The $\mathrm{THz}$ intensity is increased dramatically when the target size is increased from $200 \times 200 \mu \mathrm{m}$ to $1000 \times 1000$ $\mu \mathrm{m}$. Thereafter it becomes saturated for larger target sizes.

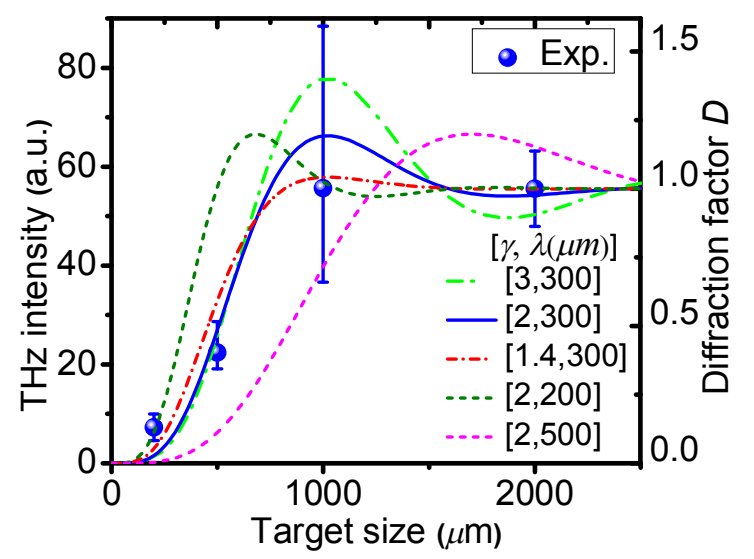


FIG. 3. Measured THz radiation intensity at $-75^{\circ}$ (blue circle) and calculated diffraction modification factor $D$ for mass-limited targets with different sizes. The solid and dashed curves are obtained with different parameters $(\gamma, \lambda)$, where $\gamma$ is the relativistic Lorentz factor of electrons and $\lambda$ the radiation wavelength in $\mu \mathrm{m}$.

The observed $\mathrm{THz}$ radiation as a function of target sizes can be explained by the diffraction effect in the model of transition radiation. The transverse extent of self-fields of relativistic electrons is $\sim \gamma \lambda$. For the finite transverse target size smaller than $\gamma \lambda$, the long-wavelength transition radiation will be significantly suppressed due to the modification of diffraction radiation. The radiation intensity from targets with finite sizes, $I_{\text {finite, }}$ can be approximately expressed as that from an infinite interface $I_{\infty}$ times a diffraction modification factor $D$. For a radiator with a finite target size of $\rho[13]$,

$D=\left[1-\frac{2 \pi \rho}{\lambda \sqrt{\gamma^{2}-1}} J_{0}\left(\frac{2 \pi \rho \sin \vartheta}{\lambda}\right) K_{1}\left(\frac{2 \pi \rho}{\lambda \sqrt{\gamma^{2}-1}}\right)-\frac{2 \pi \rho}{\left(\gamma^{2}-1\right) \lambda \sin \vartheta} J_{2}\left(\frac{2 \pi \rho \sin \vartheta}{\lambda}\right) K_{0}\left(\frac{2 \pi \rho}{\lambda \sqrt{\gamma^{2}-1}}\right)\right]^{2}$,

where $J_{\mathrm{i}}$ and $K_{\mathrm{i}}$ are respectively the $i$ th-order regular and modified Bessel functions, $\vartheta$ the angle between the observation direction and electron beam direction. Given the broadband radiation and electron energy spectra in our case, we have calculated the modification factor $D$ as a function of target sizes under different parameters, as shown by the curves in Fig. 3. With the increase of target sizes, the $\mathrm{THz}$ radiation is predicted to increase substantially till a turning point, and subsequently decrease to a constant saturation value. The corresponding target size at the turning point is mainly determined by the radiation wavelength. As shown in Fig. 3, the calculated trend with $\lambda=300 \mu \mathrm{m}$ agrees well with the experimental results.

To further clarify the radiation mechanism, we have performed experiments with different types of targets. Figure 4(a-b) shows the THz radiation from the metal-PE targets as a function of the thickness of the PE layer. The data at $0 \mu \mathrm{m}$ correspond to the pure metal foil without any PE. The THz intensity drops when there is a $15 \mu \mathrm{m}$ PE layer introduced behind the metal foil. However, when the PE thickness is increased from $15 \mu \mathrm{m}$ to $500 \mu \mathrm{m}$, the $\mathrm{THz}$ emissions increase first and then decrease, indicating that there exists an optimal PE thickness for THz generation. The optimal thickness for radiation at $75^{\circ}, 45^{\circ},-60^{\circ}$ and $-75^{\circ}$ is $57_{-17}^{+18}, 115_{-40}^{+85}, 140_{-60}^{+50}, 43_{-28}^{+27} \mu \mathrm{m}$, respectively. Even when the PE layer is up to $500 \mu \mathrm{m}$ thick, there still remains strong THz radiation. 
Cherenkov radiation could occur inside the PE layer. As a kind of bulk radiation, the Cherenkov radiation scales almost linearly with the medium thickness. Figure 4(c) shows the THz radiation from single-layer PE targets with different thickness. With the increase of PE thickness, the $\mathrm{THz}$ radiation decreases, rather than increases. This indicates that Cherenkov radiation is not the dominant contributor to the $\mathrm{THz}$ radiation.
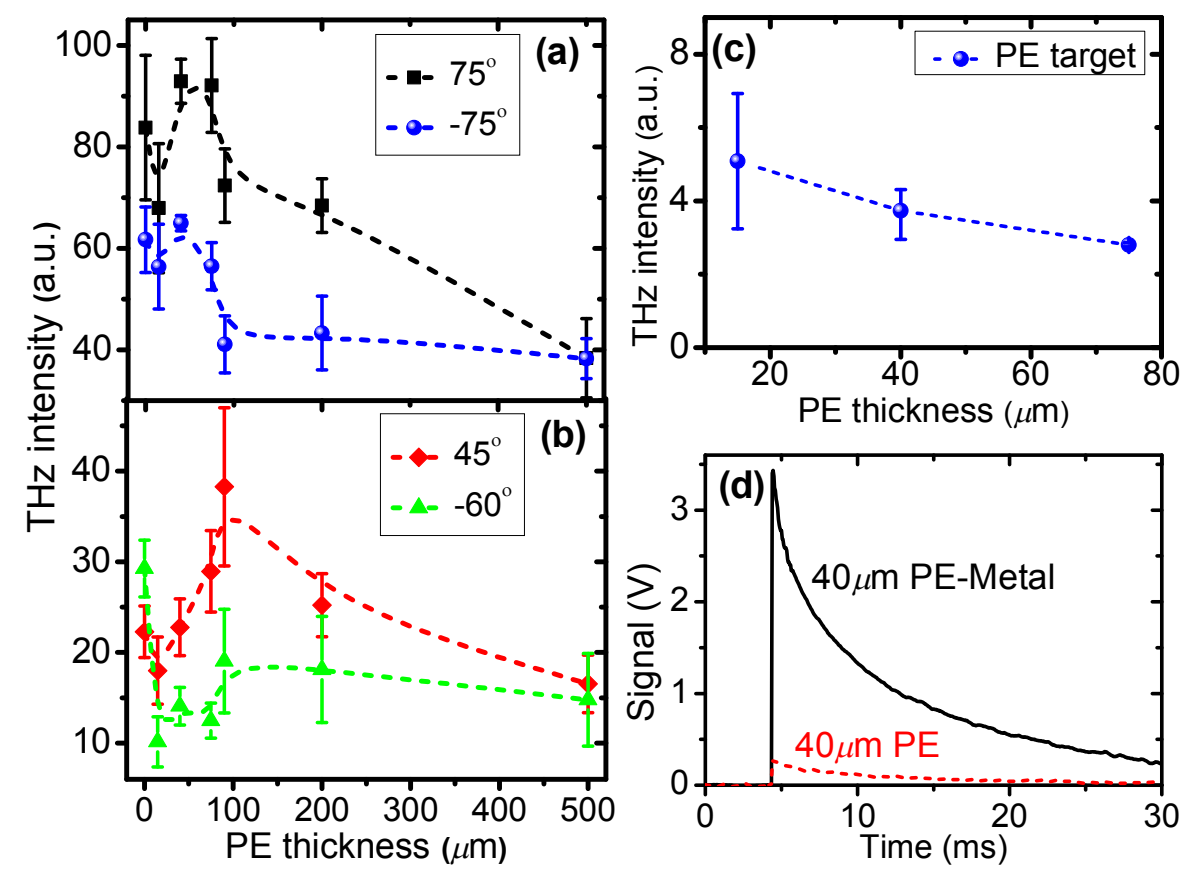

FIG. 4. (a-b) Measured THz intensity at $75^{\circ}$ (black square), $45^{\circ}$ (red diamond), $-60^{\circ}$ (green triangle) and $-75^{\circ}$ (blue circle) from the metal-PE targets as a function of the thickness of the PE layer. (c) Measured THz intensity at $-75^{\circ}$ from the single-layer PE targets with different thickness. (d) Comparison of the typical signals recorded by the $\mathrm{THz}$ detector from the single-layer PE (red dashed) and the PE-metal (black solid) targets with a $40 \mu \mathrm{m}$ PE layer.

The transition radiation depends critically on the properties of the target-vacuum boundary. To test the effect of the interface, we have ever reversed the metal-PE target and used the laser pulse to irradiate the PE layer, rather than the metal layer. Figure 4(d) shows the typical signals recorded by the $\mathrm{THz}$ detector at $-75^{\circ}$ for the reversed PE-metal and single PE targets, where both the PE layers are $40 \mu \mathrm{m}$ thick. The THz radiation from the metal boundary is about 10 times higher than that from the single PE boundary. The relative dielectric constant of the PE at the THz regime is $\sim 2.3$, while that of the metal is much greater than one [27]. The stronger emission from the metal boundary is a 
direct manifestation of transition radiation.

The features shown in Fig. 4(a-b) can be interpreted qualitatively by the formation-zone effect of transition radiation. Transition radiation is not emitted instantaneously at the interface, but occurs over a formation length [28]. For the forward radiation in the medium with a dielectric constant of $\varepsilon_{\mathrm{r}}$ observed at an angle $\vartheta$ with respect to the electron motion direction, the formation length $L_{\mathrm{f}}$ is given by

$$
L_{f}=\frac{\beta \lambda}{2 \pi\left|1-\sqrt{\varepsilon_{r}} \beta \cos \vartheta\right|},
$$

where $\beta$ is the electron velocity normalized by the light velocity in vacuum.

For the metal-PE targets, there are actually two transition radiation sources, which are located at the metal-PE interface inside the target and at the PE-vacuum interface at the rear target surface, respectively. When the thickness of the PE layer is less than $L_{\mathrm{f}}$, the forward radiation from the metal-PE interface and the backward radiation from the PE-vacuum interface will interfere with each other, thus suppressing the total forward radiation. Hence, the detected radiation drops when the $15 \mu \mathrm{m}$ PE layer is introduced behind the single metal foil. With increasing the thickness of the PE layer, the interference will disappears gradually. Consequently, the THz radiation will become stronger with the PE thickness thereafter. With the further increase of PE thickness, the detected radiation from the metal-PE interface will be reduced due to the absorption of $\mathrm{THz}$ radiation by the PE layer. On the other hand, as the electron beam propagates in the thick PE layer, the electron beam will be broadened and accordingly the beam amplitude will be decreased due to the energy dispersion. Electrons with lower energies even cannot reach to the target rear surface. Those factors will lead to a sharp drop of the radiation from the PE-vacuum interface. Therefore, there will be an optimal PE thickness. This agrees qualitatively with the observation.

The observed optimal PE thickness can be used to characterize the formation length $L_{\mathrm{f}}$. According to Eq. (2), $L_{\mathrm{f}}$ is dependent on the observation angles, i.e., the smaller the angle $\vartheta$, the larger the length $L_{\mathrm{f}}$. One can find $L_{\mathrm{f}}$ at $45^{\circ}$ and $-60^{\circ}$ is larger than that at $\pm 75^{\circ}$. This explains why the observed optimal PE thickness at $45^{\circ}$ and $-60^{\circ}$ is larger than that at $\pm 75^{\circ}$ in Figs. $4(\mathrm{a}-\mathrm{b})$. 
The THz radiation measured does not show obvious correlation with the sheath field formation and subsequent ion acceleration via TNSA. Figure 5(a) shows the ion energy spectra from the mass-limited $\mathrm{Cu}$ targets with different target sizes. When the target size is increased from $500 \times 500$ $\mu \mathrm{m}$ to $2000 \times 2000 \mu \mathrm{m}$, both the number and the maximum energy of ions are decreased substantially. A small target will confine the transverse refluxing of fast electrons, resulting in a higher sheath field for a longer time and enhanced ion acceleration [29]. But this is completely different with the $\mathrm{THz}$ radiation dependence on the target sizes shown in Fig. 3, where the THz radiation tends to become saturated as the target size is increased.
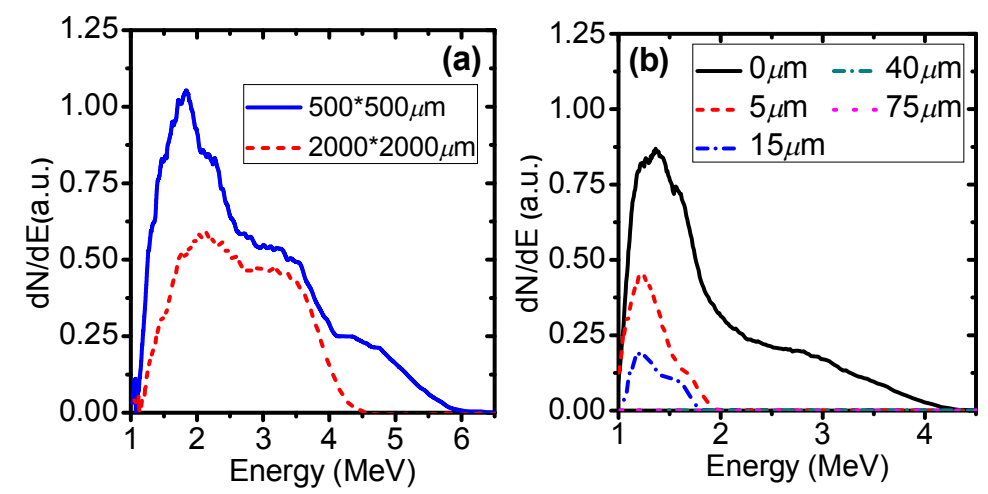

FIG. 5. (a) Measured ion energy spectra from $500 \times 500 \mu \mathrm{m}$ (blue solid) and $2000 \times 2000 \mu \mathrm{m}$ (red dashed) mass-limited $\mathrm{Cu}$ targets. (b) Ion energy spectra from the metal-PE targets with different PE thickness. The maximum energy of ions from the $40 \mu \mathrm{m}$ and $75 \mu \mathrm{m}$ thick PE layer becomes less than the lower detection limit (1 $\mathrm{MeV})$, which is caused by the shielding $\mathrm{Al}$ filters in front of IP.

Figure 5(b) shows the ion energy spectra from the metal-PE targets with different thickness of the PE layer. With the increase of PE thickness, the ion number and energy drop rapidly and even disappear for the thickness $>40 \mu \mathrm{m}$. While the THz radiation is still emitted till $500 \mu \mathrm{m}$ (see Fig. 4). As fast electrons of specific divergence angles transport through thick targets, the areal density of electrons arriving at the rear target surface is reduced. The established sheath field becomes weak, and even too weak to accelerate ions [30].

In the experiment reported by Gopal et al. [18], a correlation between the THz yield and proton 
numbers is observed by measuring their dependence on the laser energy. Such a correlation implies that the $\mathrm{THz}$ radiation and ion acceleration may be driven by a common source. Our results show that the source is the forward fast electrons. When the fast electrons pass through the rear surface-vacuum boundary of a foil, they will not only radiate $\mathrm{THz}$ radiation via transition radiation, but also set up a sheath field accelerating ions. Nevertheless, it should be noted that the laser contrast in the experiment by Gopal et al. [18] is much higher than that in our case. Despite that the $\mathrm{THz}$ radiation measured in our present experiment is mainly attributed to transition radiation, different laser-plasma conditions could result in the change of the dominated $\mathrm{THz}$ generation mechanisms $[18,19]$.

There are several evidences indicating that the measured $\mathrm{THz}$ radiation is coherent. Firstly, after taking into account both the detector responsivity and the transmittance of the optical components in the detection path, the THz energy measured at $75^{\circ}$ is $\sim 280 \mu \mathrm{J} / \mathrm{sr}$ in our experiment. It is well known that the CTR is proportional to $N_{e}^{2}$, while the incoherent transition radiation (ITR) scales with $N_{\mathrm{e}}$, where $N_{\mathrm{e}}$ is the electron number. If the radiation was generated by ITR, $N_{\mathrm{e}}$ had to be as huge as $1.75 \times 10^{20}$ to match the measured THz energy. This number is unreasonable according to the well-known conversion efficiency from the laser energy to the fast electrons. While the CTR model gives a reasonable $N_{\mathrm{e}} \sim 1.32 \times 10^{10}$. Secondly, the ITR is independent on the target size, and its spectrum is flat [13]. While in the experiment, the THz radiation is found to be strongly affected by target sizes (see Fig. 3), and the measured spectrum is frequency-dependent [see Fig. 2(a)]. Those features can be well explained by the model of CTR. Thirdly, the formation-zone effect is found with the metal-PE targets, which only occurs for coherent radiation. Those evidences suggest that the measured $\mathrm{THz}$ radiation is $\mathrm{CTR}$.

Given the experimental observation and the theoretical model of CTR, we estimate the total $\mathrm{THz}$ energy from the rear side of metal foils to be $\sim 400 \mu \mathrm{J} /$ pulse under our experimental conditions, and the corresponding energy conversion efficiency from the laser to $\mathrm{THz}$ radiation is $\sim 2 \times 10^{-4}$. The measured THz energy has approached the energy level of the state-of-the-art THz sources based on conventional linear accelerators [10]. 
In conclusion, we have demonstrated intense $\mathrm{THz}$ transition radiation of the laser-accelerated relativistic electron beams crossing the solid rear surface. The THz emissions from the mass-limited and metal-PE targets are observed to strongly depend on target parameters. It can be well explained by the model of CTR considering the effects of diffraction radiation and formation zones. The laser-plasma-based $\mathrm{THz}$ transition radiation presented could be a promising compact strong-field $\mathrm{THz}$ source. Moreover, it may provide an alternative diagnostic to infer the spatio-temporal distribution of fast electron beams generated in laser-solid interactions.

We wish to acknowledge R. Yang, X. G. Qiu at the National Laboratory for Superconductivity in Beijing for the calibration of filters. Z.M.S. acknowledges the support by a Leverhulme Trust research grant. This work is supported by the National Basic Research Program of China (2013CBA01500 and 2014CB339801), the National Nature Science Foundation of China (11520101003, 11135012, 11375262, and 11421064).

${ }^{1}$ M. Tonouchi, Nat. Photonics 1, 97 (2007).

${ }^{2}$ R. Ulbricht, E. Hendry, J. Shan, T. F. Heinz, and M. Bonn, Rev. Mod. Phys. 83, 543 (2011).

3 T. Kampfrath, K. Tanaka, and K. A. Nelson, Nat. Photonics 7, 680 (2013).

${ }^{4}$ A. J. Fitzgerald, E. Berry, N. N. Zinovev, G. C. Walker, M. A. Smith, and J. M. Chamberlain, Phys. Med. Biol. 47, R67 (2002).

5 S. Koenig et al., Nat. Photonics 7, 977 (2013).

${ }^{6}$ U. Happek, A. J. Sievers, and E. B. Blum, Phys. Rev. Lett. 67, 2962 (1991).

7 A. M. Cook, R. Tikhoplav, S. Y. Tochitsky, G. Travish, O. B. Williams, and J. B. Rosenzweig, Phys. Rev. Lett. 103, 095003 (2009).

${ }^{8}$ G. L. Carr, M. C. Martin, W. R. McKinney, K. Jordan, G. R. Neil, and G. P. Williams, Nature 420, 153 (2002).

9 Y. Shibata et al., Phys. Rev. E 52, 6787 (1995).

${ }^{10}$ Z. Wu, A. S. Fisher, J. Goodfellow, M. Fuchs, D. Daranciang, M. Hogan, H. Loos, and A. Lindenberg, Rev. Sci. Instrum. 84, 022701 (2013).

11 S. M. Hooker, Nat. Photonics 7, 775 (2013).

12 W. P. Leemans et al., Phys. Rev. Lett. 113, 245002 (2014). 
13 W. P. Leemans et al., Phys. Rev. Lett. 91, 074802 (2003); C. B. Schroeder, E. Esarey, J. van Tilborg, and W. P. Leemans, Phys. Rev. E 69, 016501(2004).

${ }^{14}$ H. Hamster, A. Sullivan, S. Gordon, W. White, and R. W. Falcone, Phys. Rev. Lett. 71, 2725 (1993).

15 A. Sagisaka et al., Appl. Phys. B 90, 373 (2008).

16 Y.-T. Li et al., Appl. Phys. Lett. 100, 254101 (2012); C. Li et al., Opt. Express 24, 4010 (2016); G.-Q. Liao et al., Phys. Plasmas 23, 013104 (2016).

17 G.-Q. Liao et al., Phys. Rev. Lett. 114, 255001 (2015).

18 A. Gopal et al., Phys. Rev. Lett. 111, 074802 (2013); A. Gopal et al., New J. Phys. 14, 083012 (2012); A. Gopal et al., Opt. Lett. 38, 4705 (2013).

19 Z. Jin, private communication.

20 W. J. Ding, Z. M. Sheng, and W. S. Koh, Appl. Phys. Lett. 103, 204107 (2013).

21 J. Zheng et al., Phys. Plasmas 9, 3610 (2002); Phys. Plasmas 10, 2994 (2003).

22 J. J. Santos et al., Phys. Rev. Lett. 89, 025001 (2002); S. D. Baton et al., Phys. Rev. Lett. 91, 105001 (2003); J. Zheng et al., Phys. Rev. Lett. 92, 165001 (2004).

23 W.-M. Wang, P. Gibbon, Z.-M. Sheng, and Y.-T. Li, Phys. Rev. E 91, 013101 (2015); M. Chen, Z. M. Sheng, J. Zheng, Y. Y. Ma, and J. Zhang, Chin. J. Comp. Phys. 25, 43 (2008) (in Chinese).

24 M. G. Haines, M. S. Wei, F. N. Beg, and R. B. Stephens, Phys. Rev. Lett. 102, 045008 (2009).

25 M. L. Ter-Mikaelian, High-Energy Electromagnetic Processes in Condensed Media, Wiley-Intersciencer, New York, 1972.

26 See Supplemental Material for more details on the estimation of electron beam divergence from the degree of $\mathrm{THz}$ radiation polarization.

27 Y.-S. Lee, Principles of Terahertz Science and Technology, Springer, New York, 2008.

28 L. C. L. Yuan, C. L. Wang, and H. Uto, Phys. Rev. Lett. 25, 1513 (1970).

29 S. Buffechoux et al., Phys. Rev. Lett. 105, 015005 (2010).

30 A. J. Mackinnon et al., Phys. Rev. Lett. 88, 215006 (2002). 